\title{
Sobrepeso y obesidad en profesores
}

\author{
Lucía Rodríguez-Guzmán ${ }^{1}$, Francisco José Díaz-Cisneros ${ }^{1}$, \\ Elizabeth Rodríguez-Guzmán ${ }^{2}$
}

Resumen

Palabras clave
Objetivo: Determinar la prevalencia de sobrepesoy obesidad en profesores de Guanajuato, México. Diseño: Estudio observacional, transversal, retrospectivo. Lugar: Instituto de Investigación sobre el Trabajo, Facultad de Medicina de la Universidad de Guanajuato. Participantes: La población de estudio consistió en 479 docentes (208 hombres y 271 mujeres). Intervenciones: Los indicadores antropométricos de peso, estatura, medición de circunferencia de cintura e índice de masa corporal fueron calculados para estimar la grasa corporal. Principales medidas de resultados: Sobrepeso y obesidad en profesores. Resultados: Las prevalencias de sobrepeso y obesidad fueron $43 \%$ y $21 \%$, respectivamente. Conclusiones: El sobrepeso y la obesidad son importantes factores de riesgo cardiovascular. Los profesores necesitan una dieta saludable y promoción de actividad física.

Obesidad; peso corporal; enfermedades cardiovasculares; docente.

\section{Overweight and obesity in teachers}

\section{Abstract}

Objetive: To determine overweight and obesity levels in teachers of Guanajuato, Mexico. Design: Observational, transversal, retrospective study. Setting: Labor Investigation Institute, Faculty of Medicine, Guanajuato University. Participants: Four hundred and seventy-nine teachers (208 men and 271 women). Interventiones: Anthropometric indicators including weight, height, waist circumference and body mass index were calculated to estimate body fat. Main outcome measures: Overweight and obesity in teachers. Results: The prevalence of overweight and obesity was $45 \%$ and $21 \%$, respectively. Conclusions: Overweight and obesity are important risk factors for cardiovascular disease. Teachers need a healthy diet and physical activity promotion.

Key words: Obesity; body weight; cardiovascular diseases; faculty.

\section{INTRODUCCIÓN}

Más de 1000 millones de adultos en todo el mundo tienen peso excesivo, y al menos 300 millones son clínicamente obesos $\left({ }^{1}\right)$. La transición nutricional que enfrentan las sociedades en desarrollo propicia un cre-

1 Instituto de Investigación sobre el Trabajo de la Universidad de Guanajuato. Guanajuato, México.

2 Facultad de Medicina de la Universidad de Guanajuato. Guanajuato, México. ciente aumento de la obesidad, hipertensión, diabetes y padecimientos cardiovasculares. Según la Organización Mundial de la Salud (OMS), las enfermedades no transmisibles se extienden con rapidez y provocan alrededor de $60 \%$ de la mortalidad mundial. Incluso entre los habitantes más pobres de las grandes ciudades, las enfermedades no trasmisibles debidas a los alimentos y hábitos malsanos coexisten con la desnutrición. Este problema de salud pública se observa desde edades tempranas de la vida $\left({ }^{2,3}\right)$ y afecta cada vez a más personas en edad productiva.

La prevalencia de obesidad es superior al $20 \%$, en 17 de los 20 países latinoamericanos, y es significativamente más alta en las mujeres. $\left({ }^{4}\right)$.

En México, la Secretaría de Salud recientemente informó en el comunicado de prensa n. 220 , que un $60 \%$ de la población mexicana presenta sobrepeso y obesidad $\left({ }^{5}\right)$. Esta declaración, sustentada en los estudios realizados por el Instituto de Investigación en Nutrición y Salud $\left({ }^{6}\right)$, confirma que dos terceras partes de las mujeres mexicanas de 18 a 49 años de edad presentan sobrepeso 
y obesidad; similar situación muestran los hombres, que en conjunto los ubica ante un importante riesgo de enfermedad.

El Tercer Panel de Expertos $\left({ }^{7}\right)$ y múltiples hallazgos referentes a padecimientos de índole cardiovascular y al síndrome metabólico en adultos $\left({ }^{8,9}\right)$, señalan que la obesidad es uno de los principales factores asociados a una extensa variedad de padecimientos. Las personas con sobrepeso u obesidad son más propensas a la hipertensión, enfermedad coronaria, de la vesícula biliar, diabetes, artritis, cáncer y apnea obstructiva $\left({ }^{10}\right)$.

La verdadera tragedia, según los señalamientos de la OMS, es que el sobrepeso, la obesidad y las enfermedades crónicas asociadas, en gran medida son prevenibles. Aproximadamente, $80 \%$ de cardiopatías, accidente vascular cerebral y diabetes tipo 2 , así como $40 \%$ de los cánceres, podrían ser evitados con una dieta saludable y actividad física regular.

En la región, se ha conducido estudios con diferentes grupos poblacionales y de trabajadores. Sin embargo, no se ha investigado al gremio más grande de América Latina: a los trabajadores docentes que cubren la importante función social de formar integralmente a las nuevas generaciones.

Con el propósito de identificar los niveles de sobrepeso y obesidad en los docentes de educación básica de Guanajuato, México, se condujo un estudio de valoración de la salud docente durante el periodo mayo-octubre de 2005.

\section{MATERIALES Y MÉTODOS}

Participaron 479 docentes (208 hombres y 271 mujeres), adscritos a escuelas ubicadas en zonas urbanas y rurales de diferentes zonas geográficas de Guanajuato. Un criterio de inclusión fue que, durante el ci- clo escolar 2005-2006 estuvieran laborando en instituciones de educación básica.

El protocolo fue avalado por el Comité de Bioética y la participación de los profesores se acordó mediante consentimiento informado y voluntario. Los docentes invitados recibieron información previa y fueron programados para la evaluación médica, que incluyó entre otras, mediciones antropométricas -peso, talla, circunferencias de cintura, cadera y muslo-, aplicadas al sujeto descalzo y con vestimenta ligera.

Para identificar el nivel de sobrepeso y obesidad, el peso fue comparado con los valores de peso ideal propuestos por la Sociedad de Actuarios Americanos $(68,8 \mathrm{~kg}$ para hombres y $55,8 \mathrm{~kg}$ para mujeres) $\left({ }^{11}\right)$, y la referencia de Casillas y Vargas $(72,9$ $\mathrm{kg}$ para hombres y 58,2 para mujeres) $\left({ }^{12}\right)$.

Según la American Heart Association, AHA $\left({ }^{13}\right)$, la circunferencia de cintura $\geq 102$ $\mathrm{cm}$ en hombres, $\mathrm{y} \geq 88 \mathrm{~cm}$ en mujeres es considerada obesidad abdominal.

El índice de masa corporal (IMC) o índice de Quetelet fue aplicado para clasificar el sobrepeso y la obesidad, a partir de la fórmula $\mathrm{IMC}=\left(\right.$ peso $\left./ \mathrm{talla}^{2}\right)=\left(\mathrm{kg} / \mathrm{m}^{2}\right) . \mathrm{La}$ OMS $\left({ }^{14}\right)$ propone IMC $\geq 25$ para riesgo moderado (sobrepeso); asimismo, IMC $\geq 30$ para riesgo cardiovascular alto (obesidad). Si el IMC es de 30 a 34,9 , se trata de obesidad I, de 35 a 39,9 , corresponde a obesidad II, y $\geq 40$ indica obesidad III. Los datos obtenidos fueron capturados en excel y se aplicó estadística descriptiva.

\section{RESULTADOS}

Las características generales del grupo de profesores estudiados muestran que la edad varía en un rango de 20 a 63 años. El promedio y la desviación estándar fueron $40,1 \pm 9$ años de edad para los hombres y $39,8 \pm 8$ años para las mujeres. 
El peso registró los siguientes promedios y desviación estándar en kilogramos: 78,7 \pm 12 y $65,9 \pm 11$, para hombres y mujeres, respectivamente. La talla mostró valores de $170,1 \pm 6$ en hombres y $157,3 \pm 6$ $\mathrm{cm}$ en mujeres.

La circunferencia de cintura, en el caso de los hombres registró una media de 95,4 $\pm 10 \mathrm{~cm}$, encontrándose que $22,5 \%$ tuvo cifras $\geq 102 \mathrm{~cm}$. Se observó diferencias significativas a mayor edad: $14,4 \%$ de los profesores de 20 a 39 años y $29 \%$ de profesores de 40 a 59 años de edad tuvieron cifras anormales. La circunferencia de cintura en las mujeres presentó una media de 92,9 \pm $14 \mathrm{~cm}, 59,6 \%$ con valores $\geq 88 \mathrm{~cm}$. En este caso, también se detectó diferencias significativas entre grupos etáreos; $45,4 \%$ de las profesoras de 20 a 39 años y $70,7 \%$ de 40 a 59 años de edad tenían circunferencia de cintura fuera del valor ideal.

El índice de masa corporal para el conjunto de docentes fue $26,9 \pm 4\left(\mathrm{~kg} / \mathrm{m}^{2}\right)$, con valores promedio de 27,2 y 26,7 , para hombres y mujeres, respectivamente.

En la Tabla 1, se observa la prevalencia de docentes con sobrepeso y grados de obesidad I, II y III, según el IMC. Se encontró que, únicamente la tercera parte del total de participantes presentaba peso normal. La prevalencia de sobrepeso fue $51 \%$ en hom-

Tabla 1. Clasificación peso, sobrepeso y obesidad en grados I, II, y III, según índice de masa corporal (IMC) en hombres y mujeres docentes.

\begin{tabular}{lrrrr}
\hline Variable & IMC & $\begin{array}{c}\text { Hombres } \\
\mathrm{n}=208\end{array}$ & $\begin{array}{c}\text { Mujeres } \\
\mathrm{n}=269\end{array}$ & $\begin{array}{r}\text { Todos } \\
\mathrm{n}=477\end{array}$ \\
\hline Delgadez & $\leq 18$ & & $0,4 \%$ & $0,2 \%$ \\
Normal & $18-24,9$ & $28,4 \%$ & $41,3 \%$ & $35,6 \%$ \\
Sobrepeso & $25-29,9$ & $51,4 \%$ & $36,0 \%$ & $43,1 \%$ \\
Obesidad I & $30-34,9$ & $15,4 \%$ & $16,3 \%$ & $16,2 \%$ \\
Obesidad II & $35-39,9$ & $4,3 \%$ & $4,5 \%$ & $4,1 \%$ \\
Obesidad III & $\geq 40$ & $0,5 \%$ & $1,5 \%$ & $1,0 \%$ \\
\hline
\end{tabular}

bres y $36 \%$ en mujeres; en tanto, la obesidad afectó a $20 \%$ de los varones y a $22 \%$ de las maestras.

\section{DISCUSIÓN}

La población estudiada era relativamente joven, con edad promedio de 40 años. La población mayor de 60 años es prácticamente escasa en este grupo de docentes. En la muestra total, el mayor porcentaje corresponde a las mujeres, lo que se explica por el hecho de que actualmente las maestras ocupan la mayoría de los puestos docentes en el nivel educativo básico.

La estatura del grupo de estudio resultó semejante a los valores de la estatura promedio para hombres y mujeres de la región. Cabe señalar que, la talla baja en México (150 cm en mujeres y $160 \mathrm{~cm}$ en hombres) parece asumir un papel predictor de riesgo de diabetes, hipertensión arterial e hipercolesterolemia. Pavía y Velásquez $\left({ }^{15}\right)$ afirman que los individuos de talla baja tienen una cantidad mayor de grasa que los individuos de talla normal, y con ello mayor riesgo de padecimientos crónicos, como diabetes y enfermedades cardiovasculares.

La comparación del peso actual con el peso ideal revela un sobrepeso de 7 a $10 \mathrm{~kg}$, tanto en hombres como mujeres del estudio, independientemente del parámetro que se seleccione. Como se discutirá más adelante, este sobrepeso se debe principalmente al nivel significativo de grasa acumulada, que coloca a la mayoría de los docentes en las categorías de sobrepeso y obesidad.

La circunferencia de cintura indica la cantidad de tejido adiposo en esa región del cuerpo y permite identificar si existe obesidad abdominal, pues el IMC no distingue entre grasa y músculo en casos específicos y en función de rasgos étnicos $\left({ }^{16}\right)$. 
Si el mayor porcentaje de la grasa corporal está localizada en la cavidad abdominal (figura de manzana con obesidad abdominal o androide), la persona tiene mayor riesgo de sufrir enfermedades metabólicas secundarias al sobrepeso: diabetes, dislipidemia, hipertensión arterial, entre otras.

Según referencias internacionales, la circunferencia de cintura $\geq 102 \mathrm{~cm}$ en un hombre $\mathrm{y} \geq 88 \mathrm{~cm}$ en una mujer, es considerada obesidad abdominal. En Europa, se propone una circunferencia de cintura $\geq 94 \mathrm{~cm}$, tanto en hombres como en mujeres. En otros países, como México, se ha encontrado que los demás elementos del síndrome metabólico están presentes, cuando la circunferencia de la cintura es mayor de 90 $\mathrm{cm}$. Es probable que esta última medida sea la más propicia para considerar la obesidad abdominal en personas de los países de América Latina.

En los resultados del presente estudio, se observa que en promedio la circunferencia de cintura en hombres $(95,4 \mathrm{~cm})$ se encuentra dentro de los límites superiores normales de los valores propuestos por la OMS y por arriba del valor propuesto para la población mexicana. En el caso de las mujeres, el promedio de la circunferencia de cintura $(92,9 \mathrm{~cm})$ es mayor a los valores de referencia nacionales e internacionales.

En la clínica, se ha tratado de simplificar el procedimiento para expresar datos indicativos de riesgo cardiovascular, por medio de la variable de circunferencia de abdomen, debido a sus elevados coeficientes de correlación con datos epidemiológicos y metabólicos. La mayor circunferencia de cintura se correlaciona, por ejemplo, con cifras elevadas de triglicéridos séricos.

La distribución del tejido adiposo en los distintos depósitos anatómicos es importante en relación con la morbilidad. En especial, la grasa intraabdominal tiene mayor valor predictivo que la grasa subcutánea de las diferentes regiones corporales.

Por otra parte, el índice de masa corporal (IMC) o índice de Quetelet, es una medición usada comúnmente para clasificar el sobrepeso y la obesidad. Referencias nacionales muestran que las cifras de riesgo cardiovascular con base en el IMC difieren de las propuestas por la OMS. Según el primer consenso mexicano para el diagnóstico y tratamiento de las dislipidemias $\left({ }^{15}\right)$, un índice menor a 23 indica riesgo cardiovascular bajo, de 23 a 26,9 , riesgo moderado, y mayor de 27 , riesgo alto.

Si se toma como parámetros tanto la propuesta nacional como la de la OMS, los resultados permiten deducir que las mujeres docentes se encuentran dentro de la categoría de riesgo moderado, con tendencia a caer en el grupo de riesgo alto; mientras que, si se considera el parámetro nacional, los hombres se encuentran dentro del grupo de riesgo por tener un promedio de IMC de 27,2.

Según dicha clasificación, del total de docentes evaluados, hombres y mujeres, solo $35,6 \%$ tiene IMC normal, es decir, el $28,3 \%$ de los hombres y $41,23 \%$ de las mujeres están dentro de los rangos normales. Se encontró una prevalencia de sobrepeso de $51,4 \%$ en hombres y de $36 \%$, en mujeres. El porcentaje de varones con obesidad corresponde a $20 \%$ y el de mujeres a $22 \%$.

En este estudio, la prevalencia de sobrepeso y obesidad en conjunto alcanza $64 \%$, dato que coincide con lo reportado para la población mexicana de similar edad. De continuar esta tendencia de incremento de peso, en una década, el $85 \%$ de toda la población estudiada tendrá sobrepeso y obesidad, empeorando de manera dramática su perfil de salud-enfermedad.

Algunos aspectos en los que se podría orientar a la población es acerca de la canti- 
dad de grasa abdominal, como indicador de complicaciones metabólicas y clínicamente más relevante que el total de tejido adiposo, definido por el índice de masa corporal (IMC); es decir, un exceso de grasa en el abdomen pronostica enfermedad cardiaca coronaria, dislipidemias, intolerancia a la glucosa, resistencia a la insulina $e$ hiperinsulinemia $\left({ }^{17,18}\right)$. Cabe recordar que, la grasa intrabdominal presenta características morfofuncionales diferentes a las de la grasa subcutánea, lo que le confiere el rasgo de riesgo alto para la salud.

El informe de expertos de la OMS $\left({ }^{19,20}\right)$ declara que la gente debería comer menos alimentos hipercalóricos, especialmente los alimentos ricos en grasa saturadas y azúcar; realizar actividad física; consumir preferentemente grasa no saturada y menos sal; ingerir frutas, hortalizas y legumbres y dar preferencia a los alimentos de origen vegetal y pescados.

La actividad física es un factor esencial para determinar el gasto energético y el control de peso. La inactividad física y el sedentarismo, independientemente de la grasa corporal, aumentan el riesgo de enfermedad cardiovascular $\left({ }^{21}\right)$.

Lo anterior justifica ampliamente la implementación de estrategias para disminuir la obesidad, mejorar la dieta y difundir talleres centrados en la práctica regular de actividad física, que promuevan oportunidades de bienestar en la población docente. Con la perspectiva de que los cambios favorables que adopten los docentes para mejorar su salud, potencialmente, los hagan extensivos a la población estudiantil que educan y al entorno de los centros escolares, se reafirma la necesidad de realizar estudios dirigidos al magisterio.

En este contexto, la práctica cotidiana de actividad física y la adecuada alimentación representan soluciones viables para disminuir la prevalencia de obesidad y sobrepeso. Tal situación amerita estrategias educativas, que pudieran ser implementadas en las escuelas, con los docentes como promotores y beneficiarios de las acciones a favor de la salud.

\section{AGRADECIMIENTO}

Se agradece el apoyo de Fondos Mixtos CONCYTEG.

\section{REFERENCIAS BIBLIOGRÁFICAS}

1. Organización Mundial de la Salud (OMS). Informe sobre la salud en el mundo. Ginebra: OMS; 2002.

2. Pajuelo J, Canchari E, Carrera J, Leguía D. La circunferencia de la cintura en niños con sobrepeso y obesidad. An Fac Med Lima. 2004;65:167-71.

3. Díaz F, Rodríguez L, Melchor T, et al. Informe del estado de salud de los estudiantes preparatorianos de la Universidad de Guanajuato. México: Universidad de Guanajuato; 2005.

4. Braguinsky J. Prevalencia de obesidad en América Latina. Anales Sis San Navarra. 2002;25:109-15.

5. Secretaría de Salud. Comunicado de prensa n. 220. México, 2006-04-19.

6. Barquera S, Tolentino L, Rivera Dommarco J. Sobrepeso y obesidad: epidemiología, evaluación y tratamiento. México: Instituto Nacional de Salud Pública; 2006.

7. ATP III. Adult Treatment Panel III. Expert Panel on Detection, Evaluation, and Treatment of High Blood Cholesterol in Adults. JAMA. 2001;285:2486-97.

8. Goodpaster BH, Krishnaswami S, Harris TB, Katsiaras A, Kritchevsky SB, Simonsick EM, et al. Obesity, regional body fat distribution, and the metabolic syndrome in older men and women. Arch Inter Med. 2005;165:777-83.

9. Carnethon MR, Loria CM, Hill JO, Sidney S, Savage PJ, Liu K, et al. Risk factors for metabolic syndrome: the coronary Artery Risk Development in Young Adulst (CARDIA) study, 1985-2001. Diabetes Care. 2004;27:2707-15.

10. American Heart Association (AHA). La obesidad/sobrepeso [monografía en Internet]. Dallas: AHA; 2006 [citado 200608-18]. Disponible en: http://www.americanheart.org

11. World Health Organization (WHO). Obesity: Preventing and managing the global epidemic. Report of a WHO Consultation on Obesity, Geneva: WHO; 1998.

12. Casillas LE, Vargas LA. Cuadros de peso y talla para adultos mexicanos. Arch Invest Med. 1980;11:157-74.

13. American Heart Association. Cifras óptimas para la salud cardíaca [monografía en Internet]. Dallas: AHA; 2006 [citado 2006-08-18]. Disponible en: http:// www.americanheart.org 
14. World Health Organization (WHO). Technical Report Series 894: Obesity: Preventing and managing the global epidemia: report of a WHO consultation. Geneva: WHO; 2000. p. 203.

15. Pavía A, Velásquez O. 1er Consenso Mexicano para el Diagnóstico y Tratamiento de las dislipidemias. México: Secretaría de Salud; 2005.

16. Eberwine D. Globesidad: una epidemia en apogeo. Revista de la Organización Panamericana de la Salud. Perspectivas de salud. 2002;7:6-11.

17. Organización Panamericana de la Salud. Las diez principales causas de muerte en países de las Américas. Tema especial. Washington; OPS; 2006.

18. Kasper LD, Fauci AS, Braunwald E, Hauser SL, Longo DL, Jameson JL, et al. Harrison: Principios de Medicina Interna. 16 ${ }^{\mathrm{a}}$ ed. Madrid: McGraw Hill; 2005.

19. Centers for Disease Control and Prevention (CDC), U.S. Chronic Disease Prevention. Physical activity and good nutrition: essential elements to prevent chronic disease and obesity. At a Glance 2006. Atlanta: CDC; 2006.
20. Nishida Ch, Uauy R, Kumanyika S, Shetty P. The joint WHO/FAO Expert Consultation on diet, nutrition and the prevention of chronic diseases: process, product and policy implications. Public Health Nutrition. 2004;7:245-50.

21. American Heart Association. Estilo de vida activo [monografía en Internet]. Dallas: AHA; 2006 [citado 200608-18]. Disponible en: http://www.americanheart.org

Manuscrito recibido el 25 de agosto de 2006 y aceptado para publicación el 21 setiembre de 2006.

\section{Correspondencia:}

Lucía Rodríguez Guzmán

Caruso 124 (116)

León Moderno, León, Guanajuato

México

C.P. 37480

Correo-e: luciarg@prodigy.net.mx 